\title{
INVERSIÓN Y DESARROLLO INDUSTRIAL, UNA APROXIMACIÓN A LA INDUSTRIA CALDENSE Y MANIZALEÑA 1985-2005
}

\author{
Edgard David Serrano Moya ${ }^{\text {i }}$, Carmen Dussán Lubert ${ }^{\mathrm{ii}}$, Oliverio Ramírez Garzón ${ }^{\text {iii }}$ \\ ${ }^{i}$ Economista y doctor en economía. Universidad de Caldas, Departamento de \\ Economía y Administración, edserrano2001@yahoo.com \\ ii Ingeniera quimica con maestría en enseñanza de las matemáticas.Universidad de \\ Caldas, Departamento de Matemáticas, depto.matematicas@ucaldas.edu.co \\ ${ }^{i i i}$ Administrador de empresas con maestría en economía. Universidad Nacional de \\ Colombia, Sede Manizales. Departamento de Administración, oramirezga@unal.edu.co
}

Investigacion

Manizales, 2008-05-02 (Rev. 2008-06-21)

\section{RESUMEN}

Este artículo es producto de la investigación "Tasa de ganancia, inversión y producción industrial en Caldas y el área metropolitana de Manizales - Villamaría, 1985 2001”(1) . En él se analiza la inversión y la toma de decisiones de inversión en la industria manufacturera en el departamento de Caldas y su área metropolitana (Manizales y Villamaría), utilizando como fuentes la Encuesta Anual Manufacturera (EAM), la Encuesta de Opinión Empresarial (EOE) y una encuesta realizada a expertos y empresas en el año 2007.

En la primera parte se realiza una presentación bastante sintética del comportamiento de la inversión en Manizales y Caldas durante el período bajo estudio, comparándolo con lo ocurrido con la inversión en Colombia; en un segundo momento se analiza el desenvolvimiento de la variable desde la EOE. En tercer lugar, se construye desde la base de diferentes fuentes teóricas y empíricas (2) la manera en que los empresarios toman decisiones en inversión, acorde con los hallazgos reportados en las entrevistas a expertos y las encuestas a empresarios de la región, constituyéndose éste último tema en un objetivo central de este artículo.

Se destaca, dentro de los hallazgos, que la inversión industrial en Caldas y Manizales no fue en el período bajo estudio una variable definitiva en el proceso de desarrollo industrial, lo que se reafirma por el bajo impacto que ésta tuvo en los sectores productivos de mayor importancia para la región y el área metropolitana. Se señala cómo la volatilidad de la inversión, la no recurrencia de la reinversión y el bajo crecimiento de las ganancias reales industriales frenaron un mejor desempeño de la industria en el ámbito del eje cafetero e impactaron la generación de empleo. Al final del período 1985-2001, el área metropolitana de Manizales había tenido una dramática caída de la inversión total industrial, tornándose negativa desde 1995, lo que no fue congruente con el comportamiento del producto bruto y el valor agregado. Así mismo, la inestabilidad de las decisiones de inversión, el crecimiento de la producción dada, la capacidad instalada no empujando la inversión y en menor grado la 
reducción en los costos laborales, determinó el camino de las ganancias y su estancamiento en las empresas de la región. La inversión perdió dinámica e incidió en que las ganancias reales industriales no crecieran y que paralelamente se produjera una caída en el stock de capital.

Desde la encuesta realizada se encontraron interesantes respuestas sobre la toma de decisiones de inversión, puesto que desde la teoría se presupone que las ganancias se utilizan para financiar la inversión y que éstas son tenidas en cuenta en el proceso de toma de decisiones para las nuevas inversiones. Pero en este caso, desde los resultados empíricos hay una relación más cercana con la teoría keynesiana de la inversión que con la kaleckiana.

\title{
PALABRAS CLAVES
}

Inversión, tasa de ganancia, toma de decisiones.

JEL: J210, J230, E220, E230.

\section{INVESTMENT AND INDUSTRIAL DEVELOPMENT, AN APPROACH TO THE CALDAS AND MANIZALES INDUSTRY 1985-2005}

\begin{abstract}
This article is the product of the research "Earning, investment and industrial production rate in Caldas and the metropolitan area of Manizales - Villamaria, 19852001". It analyzes investment and investment decision making in the manufacturing industry in the department of Caldas and its metropolitan area (Manizales and Villamaría), using sources such as Encuesta Anual Manufacturera (EAM) (Annual Manufacturing Survey) and Encuesta de Opinión Empresarial (EOE) (Enterprise Opinion Survey), and a survey carried out with experts and companies in 2007. The first part comprises a fairly synthetic presentation of investment behavior in Manizales and Caldas during the period studied, compared to what happened with investment in Colombia. In a second instance, the development of the variable from the Enterprise Opinion Survey is examined; and thirdly, the investment decision-making process in accordance to what was reported in the interviews with experts and the surveys carried out with employers in the region, built from the basis of different theoretical and empirical sources; with the latter constituting itself as the central objective of this work. Emphasis is placed on the findings that industrial investment in Manizales and Caldas was not, during the period studied, a determining variable in the industrial development process by not having the expected impact on the productive sectors of greater importance, its volatility and non-recurrence impacted the generation of employment. The non growth of real industrial earnings slowed an improved performance in the coffee axis. At the end of the 1985-2001 period, the metropolitan area of Manizales had had a dramatic drop in the total industrial investment, which had become negative since 1995, all of which was not consistent with the gross product and added value behavior. Likewise, the volatility of investment decisions, the growth of the given production, the installed capacity not pushing investment, and, to a lesser degree, the reduction in labor costs, determined the road of the profits and its stagnation in the businesses in the region. Investment lost momentum and affected the growth of
\end{abstract}


industrial real profits, as well as the fall in the capital stock. The survey carried out showed interesting responses, such as the profits being used to finance investment and that they were taken into account in the investment decision making process, creating a closer relationship with the Keynesian theory of investment than with the Kaleckian theory.

\section{KEY WORDS}

Investment, earning rate, decision-making.

\section{INTRODUCCIÓN}

El presente artículo muestra el comportamiento de la inversión industrial del área metropolitana de Manizales y el departamento de Caldas para el período 1985-2001, en un paralelo con el desenvolvimiento de la inversión en Colombia. Se destaca, dentro de los hallazgos, que la inversión industrial en Caldas y Manizales no fue en el período de análisis una variable definitiva para el desarrollo de la industria regional y local, pues el impacto esperado en los subsectores industriales de mayor importancia presentó baja correlación con la generación de empleo y con el incremento de las ganancias reales industriales, reflejándose esto en un mediocre desempeño de la industria. Al final del período 1985-2001 el área metropolitana de Manizales había tenido una caída dramática de la inversión total industrial, tornándose negativa desde 1995, lo que no fue congruente con el comportamiento del producto bruto y el valor agregado, y que se reflejó en la caída del stock de capital_(3).

Es así como la inestabilidad de las decisiones de inversión, el crecimiento de la producción por debajo de la capacidad instalada y la reducción en los costos laborales, determinó el pobre comportamiento de las ganancias y su estancamiento, que restó incentivos a los empresarios de la región.

La estructura de la inversión requiere, además, de unas condiciones en sus determinantes que estén articulados a factores económicos como la demanda y la ganancia, que motive decisiones empresariales para la acumulación de capital. La inversión pierde posibilidades de relacionarse con otras variables con efectos de encadenamiento cuando hay un alto componente importado de factores de capital, como sucedió y sucede recientemente en el caso de la industria colombiana en las últimas tres décadas. El departamento de Caldas y el municipio de Manizales no fueron ajenos al proceso.

En el artículo se analiza también la inversión utilizando la EOE, lo que permite estudiar otros aspectos que inciden en estas decisiones, los cuales son enriquecidos con un trabajo de campo a través de encuestas a empresarios y expertos en el tema. Desde la teoría de la inversión y en una perspectiva kaleckiana de la relación de ésta con las ganancias, es evidente que los empresarios pueden decidir entre consumir e invertir, pero no están en capacidad de determinar el nivel de las ganancias. Esta aproximación teórica se aleja de lo manifestado por los empresarios sobre la toma de decisiones de inversión en la industria Caldense y Manizaleña, pues desde los datos se encontró que fueron las decisiones de inversión las que determinaron las ganancias en la dinámica real. 


\section{Materiales y Métodos}

La investigación fue de tipo descriptivo. La información se obtuvo de fuentes secundarias como la Encuesta Anual Manufacturera (EAM) en el período 1985-2001 y la Encuesta de Opinión Empresarial (la cual reporta el período 1991-2006). Las cifras nominales provenientes de la EAM fueron deflactadas con el Índice de Precios del Productor -IPP-. Toda la información se encuentra a tres dígitos y para efectos de homologación el IPP tuvo como base diciembre de 1994. En el período 1985-1989, se utilizó la serie del Índice de Precios al por Mayor -IPM-, la cual se empalmó con el IPP a diciembre de 1994. Como en la Encuesta de Opinión Empresarial (EOE) se presenta una aproximación cualitativa de la visión de los empresarios frente a diferentes variables, en algunos de los análisis se utilizó el concepto de balance que corresponde a la diferencia de respuestas positivas $\mathrm{y}$ negativas frente a una pregunta determinada. Adicionalmente, para disponer de una fuente primaria en torno a la pregunta de cómo se toman las decisiones de inversión, se llevó a cabo una entrevista semiestructurada a expertos y una encuesta de carácter cualitativo a empresarios de las industrias representativas del departamento de Caldas y la ciudad de Manizales. La entrevista semiestructurada se realizó a tres expertos en administración de negocios con el objetivo de conocer sus impresiones en torno a cómo los empresarios toman decisiones de inversión (sus percepciones se resumen en el cuadro 1). La encuesta a empresarios de las industrias representativas de la ciudad de Manizales se realizó para determinar cómo toman las empresas sus decisiones de inversión, teniendo como población la información de establecimientos industriales proporcionada por Acopi para el año 2006; mediante un muestreo aleatorio simple se estuvo de acuerdo en encuestar 33 establecimientos en funcionamiento, de los cuales únicamente 23 mostraron disposición para participar de la investigación. La encuesta se realizó entre los meses de julio y agosto de 2007, se trabajó a un nivel de significancia del 5\% y con un error del $7 \%$.

\section{Resultados y Discusión}

\section{Comportamiento de la inversión en Manizales y Caldas.}

Durante la década de los ochenta en Caldas y Manizales, la inversión en algunos sectores se benefició de la revaluación del peso, al abaratar ésta la importación de maquinaria y equipo en el período 1985-1990. En un intervalo más largo de análisis 1980-1991, la inversión mostró una alta variabilidad, donde aspectos como los cambios del entorno y el ciclo industrial influyeron en este comportamiento. Durante este período el punto más alto de la inversión según los datos de la EAM se situó en 1982 con un crecimiento del 278\%, y el más bajo en 1984 con una disminución del 94\%. La inversión neta cayó en $-40.3 \%$ entre 1980 y 1991, la variación de la inversión fue negativa y decreció a un ritmo de $-4.77 \%$ en estos años. En el caso de Colombia, en 1984 la inversión neta cayó en ese entorno de crisis económica, pero con las políticas tomadas por el gobierno buscando una reactivación, ésta se incrementó hasta 1990 cuando de nuevo la tendencia se revirtió (3). Según Lotero (6), el crecimiento del Stock de capital en el departamento de Caldas fue elevado, 18.6\% anual para el período 19751989, inversión que se concentró en la ciudad de Manizales. Lotero (6) encontró que el indicador de productividad aparente (valor agregado por trabajador) mostraba notables incrementos a finales de los años ochentas. Sin embargo, como lo considera el trabajo de Bonilla (1) este no es un indicador completo del 
progreso técnico, siendo el indicador de Productividad Total de los Factores (PTF) más confiable. Según la literatura especializada, el cambio técnico no había contribuido de manera significativa al crecimiento industrial de Caldas en la época (1), un resultado que resultaba extrapolable a Manizales.

En los años ochenta la inversión industrial en el área metropolitana de Manizales tuvo una participación del 2\% en el agregado nacional, siendo los años 1985 y 1989 los de mayor dinamismo inversor (figura 1), y los sectores textil y de alimentos los que más aportaron a este dinamismo.

En la década de los noventa la relación antes analizada mostró una alta volatilidad y la participación del área metropolitana de Manizales para 1992 decreció al 1.1\%, siendo positivo hasta 1995 -este año mostró un alto volumen al igual que lo sucedido en el ámbito nacional- y, a partir de allí, tuvo un comportamiento negativo, el cual no había logrado revertirse por lo menos hasta el 2001. Para esta década el sector textil prácticamente desaparece del escenario industrial de la región (7) y la inversión neta total fue negativa; las empresas representativas entran en concordato, y es el sector de mayor expulsión de trabajadores presentándose allí un claro proceso desindustrializador $(8)$.

En el lapso 1995-1999 se presentó a nivel nacional el mayor cierre de empresas de la época, hecho que también se presentó en Caldas y en particular en Manizales y su área metropolitana, con un apreciable número de establecimientos disueltos (7). Lo que se correlacionó con la dinámica de la inversión y las percepciones de los representantes de las industrias en la Encuesta de Opinión Empresarial, tal como se analizará más adelante.

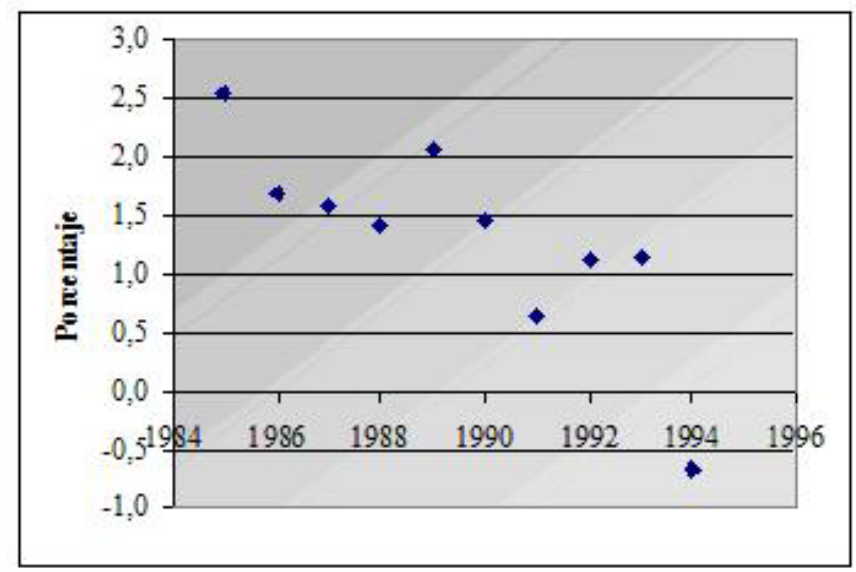

Fuente: Grupo de investigación 2007 con base en EAM 1985 - 2001

Comportamiento de la Inversión desde la EOE. La tabla 1 presenta una síntesis de la opinión de los empresarios frente al comportamiento económico del producto más representativo de cada empresa entre los años 1991-2006 (4). En 1991 las expectativas eran altas, tanto que el balance (5) fue bastante positivo, pero a partir de 1994 con el balance más alto de los noventa, la perspectiva de los empresarios cambia su tendencia 
y empieza a deteriorarse, coincidiendo con el punto de quiebre de la inversión y del Stock de capital en el análisis de la EAM.

\begin{tabular}{|c|c|c|c|c|c|c|c|}
\hline \multirow[b]{2}{*}{ Opiniones } & \multicolumn{7}{|c|}{ Año } \\
\hline & 1991 & 1994 & 1996 & 1999 & 2002 & 2005 & $2006 \%$ \\
\hline Balance & 50 & 59 & 13 & -13 & 27 & 57 & 58 \\
\hline Buena & & & 33 & 8 & 32 & 61 & 62 \\
\hline Aceptable & & & 47 & 71 & 63 & 35 & 34 \\
\hline Mala & & & 20 & 21 & 5 & 4 & 4 \\
\hline
\end{tabular}

Fuente: CRECE, EOE, FEDESARROLLO. Diciembres /*julios.

La situación se mostró claramente desfavorable desde 1996 para los empresarios de la industria, a esto se le suma que desde 1991 se veía en la percepción de diferentes sectores económicos un deterioro por la baja demanda, la lenta rotación de cartera, los altos costos financieros y el contrabando y, en menor grado, el difícil acceso al crédito bancario (EOE). Las expectativas de los empresarios cambian a partir del año 2002, las percepciones frente al producto en la industria local y regional varían favorablemente expresándose en el indicador de Balance de la EOE con calificaciones cercanas a las del primer lustro de finales de los noventa (tabla 1).

Por otra parte y como se observa en la tabla 2, hasta 1996 la apreciación sobre la capacidad de producción que tenían los empresarios sobre su organización era positiva; entre los años 1997 y 2004 la actitud pasó de una visión de aumento en la capacidad de producción de la empresa a una de mantenimiento de la misma, aspecto que se relaciona con las tendencias descritas en la EAM. A partir del 2005 se percibe por un buen grupo de los empresarios que la capacidad de producción aumenta.

\begin{tabular}{|c|c|c|c|c|c|c|c|c|c|c|c|c|c|c|c|c|}
\hline & \multicolumn{16}{|c|}{ Año } \\
\hline & 1991 & 1992 & 1993 & 1994 & 1995 & 1996 & 1997 & 1998 & 1999 & 2000 & 2001 & 2002 & 2003 & 2004 & 2005 & 2006 \\
\hline Balance & 54 & 41 & 45 & 45 & 59 & 42 & 6 & 8 & 6 & 8 & 38 & 15 & 9 & 14 & 47 & 65 \\
\hline Aumentó & & & & & 59 & 50 & 28 & 23 & 31 & 25 & 45 & 30 & 23 & 14 & 53 & 65 \\
\hline Se mantuvo & & & & & 41 & 42 & 50 & 62 & 44 & 58 & 48 & 55 & 64 & 86 & 41 & 35 \\
\hline Disminuyó & & & & & $\mathbf{0}$ & 8 & 22 & 15 & 25 & 17 & 7 & 15 & 14 & $\mathbf{0}$ & 6 & $\mathbf{0}$ \\
\hline
\end{tabular}

Fuente: CRECE, EOE, FEDESARROLLO. Eneros.

En la tabla 3 se mencionan los factores que afectan la inversión de los empresarios. En 1991 el ítem "escasez de crédito interno", que era el más importante para los empresarios, pasó a un segundo plano en la percepción que estos tienen en los siguientes años, y el "bajo nivel de demanda" se torna en el elemento clave a partir de 
1996. Para el año 2002, los problemas de baja demanda se mostraban aún como un determinante de la inversión, persistiendo esta apreciación hasta el 2006, aunque en el año 2004 la escasez de crédito externo e interno y de recursos financieros propios fueron un impedimento importante en la visión de los empresarios.

\begin{tabular}{|c|c|c|c|c|c|c|}
\hline & \multicolumn{6}{|c|}{ Año } \\
\hline & 1991 & 1996 & 1999 & 2002 & 2004 & 2006 \\
\hline $\begin{array}{l}\text { Bajo nivel de la } \\
\text { demanda interna }\end{array}$ & 21 & 29 & 35 & 55 & 25 & 27 \\
\hline $\begin{array}{l}\text { Falta de incentivos } \\
\text { para las } \\
\text { exportaciones }\end{array}$ & 3 & 9 & 7 & 10 & 0 & 13 \\
\hline $\begin{array}{l}\text { Protección } \\
\text { inadecuada frente } \\
\text { a las } \\
\text { importaciones }\end{array}$ & 2 & 11 & 7 & 0 & 0 & 13 \\
\hline $\begin{array}{l}\text { Dificultad para la } \\
\text { importación de } \\
\text { maquinaria y } \\
\text { equipo }\end{array}$ & 3 & 0 & 0 & 0 & 8 & 0 \\
\hline $\begin{array}{l}\text { Relación precio- } \\
\text { costo } \\
\text { insatisfactorio }\end{array}$ & 11 & 0 & 4 & 5 & 0 & 7 \\
\hline $\begin{array}{l}\text { Escasez de } \\
\text { insumos }\end{array}$ & 5 & 3 & 2 & 5 & 0 & 7 \\
\hline $\begin{array}{l}\text { Escasez de } \\
\text { personal adecuado }\end{array}$ & 5 & 0 & 0 & 0 & 0 & 0 \\
\hline $\begin{array}{l}\text { Escasez de crédito } \\
\text { interno }\end{array}$ & 28 & 6 & 11 & 5 & 17 & 0 \\
\hline $\begin{array}{l}\text { Escasez de crédito } \\
\text { externo }\end{array}$ & 1 & 3 & 0 & 5 & 25 & 0 \\
\hline $\begin{array}{l}\text { Escasez de } \\
\text { recursos } \\
\text { financieros } \\
\text { propios }\end{array}$ & 17 & 14 & 7 & 10 & 17 & 7 \\
\hline $\begin{array}{l}\text { Costos financieros } \\
\text { desfavorables }\end{array}$ & 7 & 17 & 20 & 0 & 0 & 0 \\
\hline $\begin{array}{l}\text { No hay } \\
\text { innovaciones } \\
\text { tecnológicas }\end{array}$ & 1 & 3 & 0 & 0 & 8 & 0 \\
\hline
\end{tabular}




\begin{tabular}{|l|c|c|c|c|c|c|}
\hline $\begin{array}{l}\text { Dificulta } \\
\text { organizacionales } \\
\text { para implementar } \\
\text { los cambios }\end{array}$ & 3 & 3 & 0 & 6 & 0 & 7 \\
\hline Altos impuestos & 5 & 3 & 10 & 9 & 0 & 20 \\
\hline
\end{tabular}

Fuente: CRECE, EOE, FEDESARROLLO. Eneros.

El panorama económico industrial registró un balance negativo durante todo $1996 \mathrm{y}$ sólo hasta el primer trimestre de 1997 dio visos de una tenue recuperación, para luego entrar en un proceso de recesión. La contracción del mercado regional repercutió en el mercado laboral, la flexibilización del mercado favoreció los despidos, perdiéndose 2.273 empleos en el sector industrial (83.65\% del desempleo generado en su momento) (Cámara de Comercio Manizales, 1997).

La percepción sobre el aumento de la capacidad productiva para algunos años en el período 1991-2006 se observa en la tabla 4, allí los empresarios entrevistados se inclinaron por ser moderados en su predicción de aumento en la capacidad instalada; los que consideraron ésta superior al $50 \%$ fueron muy pocos en todo el período, reflejando en general escepticismo por el crecimiento productivo.

Tabla 4. Percepción de aumento en la capacidad de

Producción de la empresa 1991-2006

\begin{tabular}{|c|c|c|c|c|c|c|c|c|}
\hline \multicolumn{1}{|c|}{ Año } & \multicolumn{3}{|c|}{} \\
\hline \hline & $\mathbf{1 9 9 1}$ & $\mathbf{1 9 9 6}$ & $\mathbf{1 9 9 9}$ & $\mathbf{2 0 0 2}$ & $\mathbf{2 0 0 4}$ & $\mathbf{2 0 0 6}$ \\
\hline $\begin{array}{c}\text { Entre } \\
1 \mathrm{y} \\
20 \%\end{array}$ & 29 & 57 & 69 & 43 & 100 & 45 \\
\hline $\begin{array}{c}\text { Entre } \\
\begin{array}{c}21 \mathrm{y} \\
50 \%\end{array}\end{array}$ & 15 & 32 & 16 & 43 & 0 & 45 \\
\hline $\begin{array}{c}\text { Más } \\
\text { de } \\
50 \%\end{array}$ & 0 & 13 & 15 & 14 & 0 & 10 \\
\hline
\end{tabular}

Fuente: CRECE, EOE, FEDESARROLLO. Eneros.

En general, la percepción frente a la capacidad de producción mostrada entre $1 \%$ y $20 \%$ en todo el período de análisis es recurrente. Ante una demanda por bienes industriales deprimida en la época de crisis, el volumen de pedidos cae, generando aumento en los inventarios. La respuesta de los empresarios a esta situación fue mantener la actividad productiva de las empresas, lo que se corrobora desde la EAM con el mantenimiento de la producción en el período 1991-1999, y con la caída de la inversión y el empleo. Con relación al principal tipo de inversión realizada por las empresas, es destacable cómo en plena crisis (1999) la inversión en reposición de maquinaria y equipo fue 
importante para un número considerable de estas empresas, aunque no se efectúa tal inversión como se mencionó por los hallazgos desde la EAM. Desde el año 2003 la expansión de la capacidad instalada se tornó en el principal tipo de inversión (tabla 5), las empresas líderes de la industria empezaron a ampliarse ante las nuevas expectativas generadas en el mercado interno y externo a partir del año 2003. En el año 2002 se introdujeron nuevos procesos sin necesidad de ampliación.

\begin{tabular}{|l|l|l|l|l|l|l|l|l|l|l|l|}
\hline \multicolumn{7}{|c|}{ Tabla 5. Principal tipo de inversión realizada el año } \\
anterior 1991-2006
\end{tabular}

Fuente: CRECE, EOE, FEDESARROLLO. Eneros.

A finales de la década de los noventa la situación económica de Manizales y Caldas se sintetiza en una percepción crítica por parte de los empresarios, aunque contradictoria frente a los hechos, pues se produjeron aumentos de producción (EAM) y se mantuvo la visión de capacidad de producción. Sin embargo, es innegable la desaceleración de la economía regional acentuando el problema con la crisis internacional, el contrabando y la violencia como un fenómeno que iba en aumento en la región. La evidencia empírica (EAM) muestra como el debilitamiento de la inversión en Caldas para la segunda mitad de la década del noventa, coincide con una tendencia decreciente en la percepción de la capacidad instalada promedio de la industria; por otra parte, no se presenta relación entre la inversión y los incrementos en la producción.

\section{Sobre las decisiones de inversión. Una mirada empírica.}

Se ha afirmado que el papel del empresario es clave en la toma de decisiones para la inversión. Desde esta perspectiva, los empresarios están motivados por incentivos económicos en la toma de decisiones, pero también hay una serie de variables de 
carácter no económico que inciden en estas decisiones y que es interesante tenerlas presentes en los análisis económicos. Las preguntas en torno a cómo se establecen las decisiones de inversión implican una serie de elementos que, al ser establecidos de forma esquemática (diagrama 1), evidencian la complejidad del proceso.

Diagrama 1. Sobre las decisiones de inversión

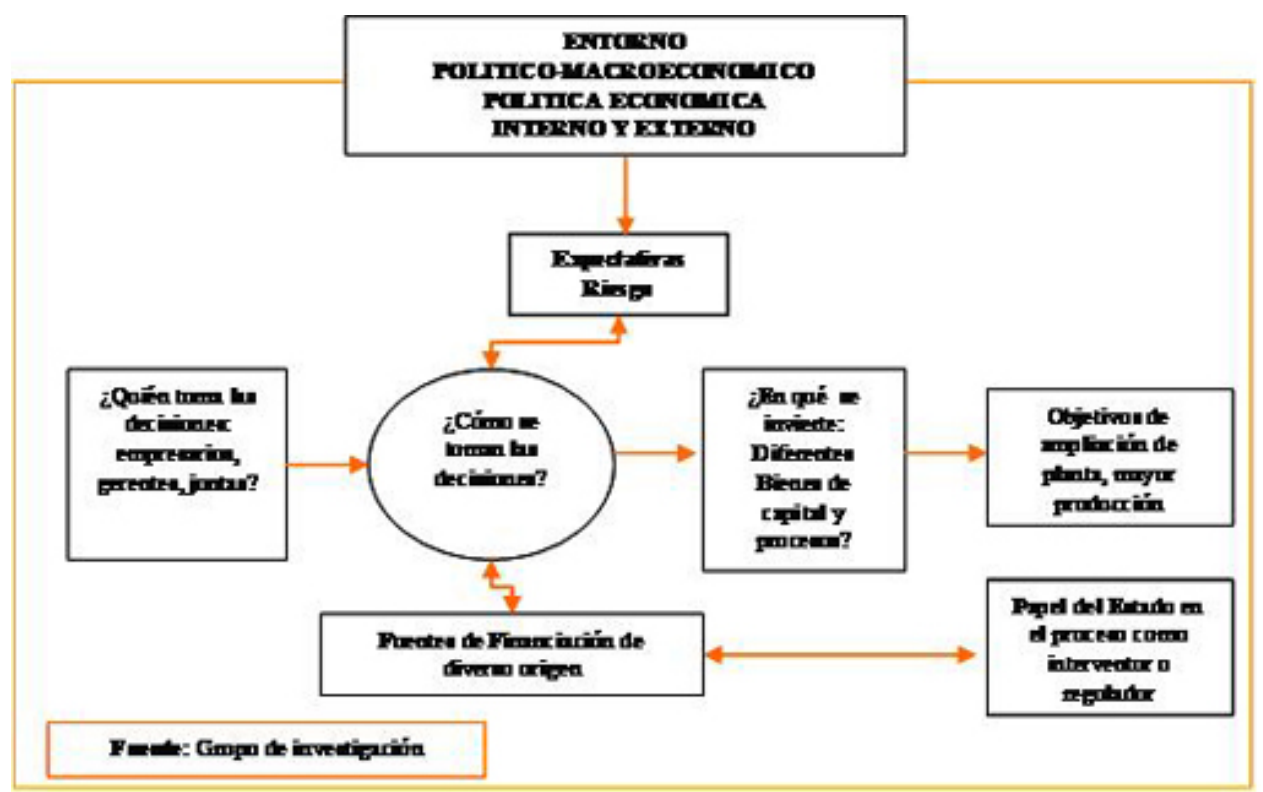

Acorde con la información recopilada de fuentes primarias $\underline{(6)}$ y secundarias (EOE) para el caso de la industria de Manizales, las motivaciones y la toma de decisiones de inversión de los agentes involucrados en estas decisiones se pueden sintetizar en el cuadro 1 .

\begin{tabular}{|c|c|c|c|c|c|}
\hline \multicolumn{6}{|c|}{ Cuadro 1. Síntesis sobre toma de decisiones de inversión } \\
\hline $\begin{array}{c}\text { FUENT } \\
\text { E }\end{array}$ & $\begin{array}{c}\text { DECISO } \\
\mathbf{R}\end{array}$ & $\underset{\mathbf{N}}{\text { MOTIVACIÓ }}$ & $\begin{array}{l}\text { TIPO DE } \\
\text { INVERSIÓ } \\
\text { N }\end{array}$ & OBJETIVO & $\begin{array}{c}\text { EXPECTATIVA } \\
\mathbf{S}\end{array}$ \\
\hline Expertos & $\begin{array}{c}\text { Empresari } \\
\text { o }\end{array}$ & $\begin{array}{l}\text { Intuición, } \\
\text { experiencia y } \\
\text { ganancia }\end{array}$ & $\begin{array}{l}\text { Equipamient } \\
\text { o industrial y } \\
\text { planta }\end{array}$ & $\begin{array}{l}\text { Supervivenci } \\
\text { a de la } \\
\text { empresa o } \\
\text { mayores } \\
\text { ganancias }\end{array}$ & $\begin{array}{l}\text { Reducción de } \\
\text { costos }\end{array}$ \\
\hline Encuesta & $\begin{array}{c}\text { Empresari } \\
\text { o }\end{array}$ & $\begin{array}{l}\text { Instinto, } \\
\text { experiencia y } \\
\text { ganancia }\end{array}$ & $\begin{array}{l}\text { Equipos y } \\
\text { ampliación } \\
\text { de planta }\end{array}$ & $\begin{array}{l}\text { Recuperación } \\
\text { de inversión, } \\
\text { ganancias, } \\
\text { permanecer } \\
\text { en el } \\
\text { mercado }\end{array}$ & $\begin{array}{c}\text { Mayores } \\
\text { ganancias y } \\
\text { recuperación de la } \\
\text { inversión }\end{array}$ \\
\hline $\mathrm{EOE}$ & $\begin{array}{c}\text { Empresari } \\
\mathrm{o}\end{array}$ & $\begin{array}{l}\text { Influencia de } \\
\text { las condiciones }\end{array}$ & $\begin{array}{c}\text { Reposición } \\
\text { de }\end{array}$ & $\begin{array}{l}\text { Aumento en } \\
\text { la capacidad }\end{array}$ & $\begin{array}{l}\text { Ampliación de } \\
\text { mercado }\end{array}$ \\
\hline
\end{tabular}




\begin{tabular}{|c|c|c|c|c|}
\hline & & $\begin{array}{l}\text { económicas y } \\
\text { políticas en la } \\
\text { toma de } \\
\text { decisiones, alto } \\
\text { nivel de la } \\
\text { demanda } \\
\text { interna, } \\
\text { disponibilidad } \\
\text { de recursos de } \\
\text { inversión } \\
\text { propios* }\end{array}$ & $\begin{array}{l}\text { Maquinaria o } \\
\text { equipo, } \\
\text { ampliación } \\
\text { de la } \\
\text { capacidad } \\
\text { instalada }\end{array}$ & $\begin{array}{c}\text { de } \\
\text { producción }\end{array}$ \\
\hline
\end{tabular}

* Se toma como referencia las respuestas favorables entre 2002 y 2006.

Al contrastar la información recopilada en la encuesta, la EOE y las entrevistas a expertos, con los elementos teóricos expuestos, se encuentra que existe consenso al tomar decisiones de inversión en las respuestas de los expertos y las consignadas en la encuesta, mientras que frente a la EOE algunas de las motivaciones son distintas. De acuerdo a las entrevistas a los expertos, las decisiones de inversión se toman con base en la experiencia de los empresarios o sus gerentes y la intuición influye en esa toma de decisiones; en las medianas empresas se planean los procesos de inversión y en las pequeñas empresas se trabaja por instinto en una lucha del "día a día". Para los expertos, las decisiones de inversión se ven influenciadas por las ganancias, como se prevé desde la perspectiva teórica keynesiana, aunque no kaleckiana, lo que según ellos sucede en los países industrializados en donde dicha decisión se encuentra también determinada por el factor tiempo, en tanto se puede dar que se quieran altas ganancias en el corto plazo o pequeñas en el largo plazo. Se argumenta por los expertos (7) que no hay un buen conocimiento del mercado interno por parte de los empresarios colombianos y que falta mayor motivación para la creación de nuevos productos para nuevos mercados, aspectos ligados directamente con la toma de decisiones.

De acuerdo a la encuesta realizada en la investigación, es claro cómo el "instinto" (8) influye en la toma de decisiones para los negocios, pero el $83 \%$ de los empresarios igualmente consideró la tasa de ganancia como uno de los aspectos que determina las decisiones de inversión. En este sentido en las diferentes empresas encuestadas de la ciudad de Manizales, se puede concluir que las decisiones de inversión que se toman en las empresas se ven determinadas por la tasa de ganancia. Pero las empresas recurren a la reinversión de sus ganancias $(26 \%)$ y mucho menos a los créditos (13\%) para financiar la inversión, aunque el $61 \%$ respondió que utilizaba ambas formas. Al analizar la información consignada en la EOE, el acceso al crédito nunca fue un obstáculo definitivo en las decisiones de inversión (tabla 3) aunque entre 1991 y 1995 pudo incidir en la toma de decisiones. Lo que se considera un hallazgo importante pues en el país siempre se ha argumentado que el acceso al crédito interno es un factor que no apoya la inversión para cualquier actividad económica.

Por otra parte el destino de las inversiones se concentró en el inicio de los noventas en la reposición de maquinaria y equipo (tabla 5). Lo que también se aprecia en la encuesta realizada a las empresas, donde el $48 \%$ de ellas manifestó que la inversión en este ítem 
ha sido importante, aunque se destacó que es la ampliación en capacidad instalada el foco de la inversión desde el año 2002.

Cuando se analiza la EOE en la búsqueda de aspectos diferentes a los económicos que incidan en las decisiones de inversión, se observa como en momentos de crisis política del país (tabla 4) las decisiones se encuentran permeadas por la percepción que tienen los empresarios y sus gerentes de la situación económica del país, y sobre todo, de la situación política y social.

Así mismo, son importantes los factores asociados a variables macroeconómicas como la demanda (tabla 2) y la actividad productiva, pero se observa una fuerte relación entre el comportamiento inversor y las apreciaciones que sobre el entorno tienen los empresarios. Dando peso a la hipótesis sobre cómo influye la inestabilidad/estabilidad política en la inversión industrial (9) y qué se puede sustentar en los recientes aportes de la teoría institucionalista, se percibe la necesidad de tener instituciones fuertes y creíbles como motivación para que los empresarios desarrollen inversiones.

\begin{tabular}{|l|l|l|l|l|l|l|l|l|l|}
\hline Tabla 6. Opinión sobre las condiciones económicas actuales \\
para la inversión \\
\hline & Año \\
\hline Opiniones & $\mathbf{1 9 9 2}$ & $\mathbf{1 9 9 4}$ & $\mathbf{1 9 9 6}$ & $\mathbf{1 9 9 8}$ & $\mathbf{2 0 0 0}$ & $\mathbf{2 0 0 2}$ & $\mathbf{2 0 0 5}$ & $\mathbf{2 0 0 6}$ \\
\hline Balance & 15 & 5 & -70 & -76 & -34 & 0 & 20 & 45 \\
\hline Favorables & & 33 & 0 & 3 & 14 & 20 & 40 & 54 \\
\hline Neutras & & 39 & 30 & 18 & 38 & 60 & 40 & 38 \\
\hline Desfavorables & & 28 & 70 & 79 & 48 & 20 & 20 & 9 \\
\hline
\end{tabular}

Fuente: CRECE, EOE, FEDESARROLLO. Noviembres.

Lo anterior es claro cuando se analiza cómo en los años noventa se presentó una tendencia que relacionaba el comportamiento de la inversión y la deplorable situación política que enfrentó el país (figura 2 y tablas 6 y 7), encontrándose que la respuesta a la inversión ante tales eventos fue negativa en el balance luego del año 1996 hasta el año 2001, situación que se expresó en la caída de la inversión en la industria y en los diferentes sectores. 
Figura 2. Relación de la inversión con las percepciones de situación política y económica en la EOE 1992 - 2000

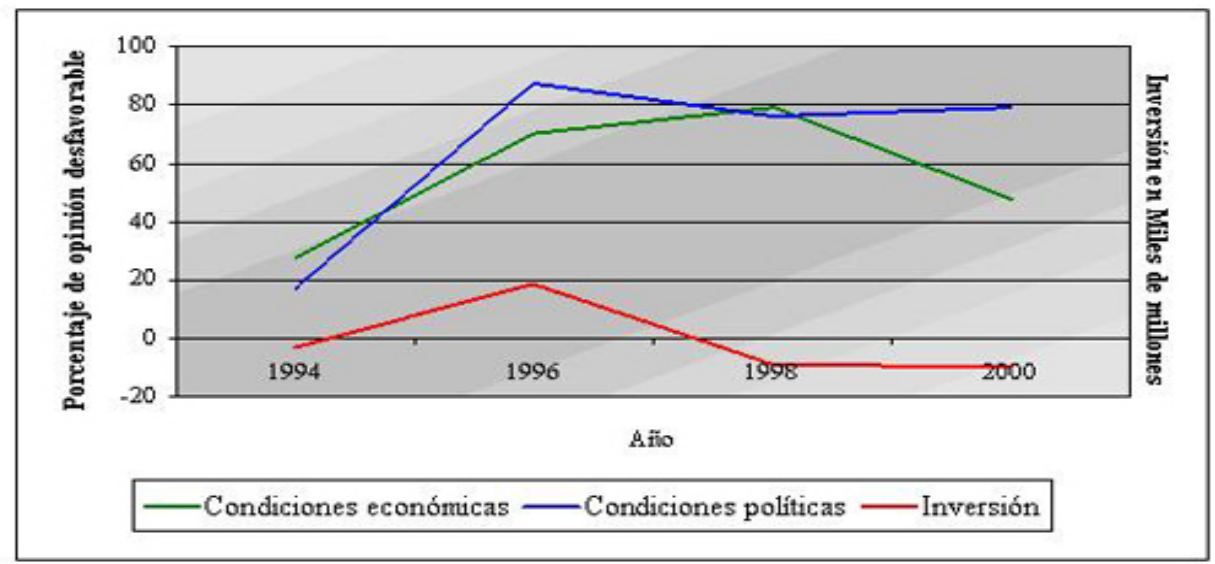

Fuente: grupo de investigación, con base en la Encuesta de Opinión Empresarial.

Es evidente cómo la situación económica y política que cambió a partir del año 2002 generó un cambio en la tendencia de la inversión, lo que se relacionó adicionalmente con el buen ambiente inversor que se tuvo para la primera mitad de la presente década en la industria regional, que se puede corroborar además por las respuestas de aumento de la inversión en la EOE entre 2002 y 2006. Al analizar la información sobre la inversión industrial y relacionarla con la situación política (tabla 7), ésta presentó una caída importante en las respuestas favorables desde el año 1996 y sólo una mejora desde 2002 (9).

\begin{tabular}{|l||c|c|c|c|c|c|r|r|r|r|}
\hline \multicolumn{1}{|c|}{ Tabla 7. Inversión y la situación política y social actual } \\
\hline \multicolumn{1}{|c|}{ Año } \\
\hline Opiniones & $\mathbf{1 9 9 2}$ & $\mathbf{1 9 9 4}$ & $\mathbf{1 9 9 6}$ & $\mathbf{1 9 9 8}$ & $\mathbf{2 0 0 0}$ & $\mathbf{2 0 0 2}$ & $\mathbf{2 0 0 5}$ & $\mathbf{2 0 0 6}$ \\
\hline Balance & -27 & 5 & -87 & -70 & -79 & 14 & 32 & 30 \\
\hline Favorables & & 22 & 0 & 6 & 0 & 43 & 42 & 43 \\
\hline Neutras & & 61 & 13 & 18 & 21 & 29 & 47 & 44 \\
\hline Desfavorables & & 17 & 87 & 76 & 79 & 29 & 11 & 13 \\
\hline
\end{tabular}

Fuente: CRECE, EOE, FEDESARROLLO. Noviembres.

\section{CONCLUSIONES}

- La inversión industrial en Caldas y Manizales no fue, en el período de estudio, una variable definitiva en el proceso de desarrollo industrial al no tener el impacto esperado en los sectores productivos de mayor importancia, su volatilidad y no recurrencia impactaron la generación de empleo, y el no crecimiento de las ganancias reales industriales frenaron un mejor desempeño en el ámbito del eje cafetero. Al final del período 1985-2001 el área metropolitana de Manizales había tenido una caída de la inversión total industrial dramática, tornándose negativa desde 1995, lo que no fue congruente con el comportamiento del producto bruto y el valor agregado. 
- La inestabilidad de las decisiones de inversión, el crecimiento de la producción dada, el uso de la capacidad instalada por debajo de la misma (no empujó la inversión) y en menor grado la reducción en los costos laborales, determinó el camino de las ganancias y su estancamiento en las empresas de la región. La inversión perdió dinámica e incidió en que las ganancias reales industriales no crecieran y que paralelamente se produjera una caída en el stock de capital.

- El mantener una alta tasa de inversión es clave en el contexto de la industria, la estructura de la inversión requiere de unas condiciones en sus determinantes que están articulados a factores económicos como la demanda y la ganancia, que motive decisiones empresariales de acumular capital. La inversión global pierde en efectos de encadenamiento (5) cuando hay un alto componente importado como ha sucedido en el caso de la industria colombiana en las últimas tres décadas, y Caldas y Manizales no fueron ajenas al proceso.

- Desde la teoría de la inversión y en una perspectiva kaleckiana de la relación de ésta con las ganancias, es evidente que los empresarios pueden decidir entre consumir e invertir pero no están en capacidad de determinar el nivel de las ganancias. Esta aproximación teórica se acerca más a la descripción de los hechos en la industria caldense y manizaleña, pues son las decisiones de inversión las que determinaron las ganancias.

- Es útil el análisis del CID (2) sustentado en los argumentos de Kaldor (4), puesto que allí se destaca a la industria como motor de crecimiento y, desde su trabajo empírico, se confirma que la tasa de crecimiento del PIB departamental aumentará $5 \%$ si la tasa de crecimiento del producto industrial es del $7.2 \%$, pero si el producto industrial no crece se produce una rápida disminución de la participación del producto industrial en el PIB, aspecto que en nuestro trabajo para Caldas y Manizales se ha detectado, teniendo como consecuencia que una menor participación del PIB industrial en el PIB nacional se relaciona con una disminución del ritmo de crecimiento de la producción y de la inversión industrial.

- Desde la encuesta realizada se encontraron interesantes respuestas en cuanto que las ganancias se utilizaban para financiar la inversión y que éstas eran tenidas en cuenta en el proceso de toma de decisiones para la inversión, dándose desde allí una relación más cercana con la teoría keynesiana de la inversión que con la kaleckiana.

\section{BIBLIOGRAFÍA}

- Bonilla, M. Tendencias de la productividad en la industria manufacturera colombiana (1974-1989). En Estrategia Industrial e inserción internacional. Luis J. Garay Editor. Fescol. 1992.

- CID, Bienestar y Macroeconomía 2002-2006. Unal-CID OCSE y CGR. Bogotá. 2006.

- García, J G. Liberación, cambio estructural y crecimiento económico en Colombia. En: Cuadernos de Economía No. 36. U.N. CID. Escuela de Economía. 2002. 
- Kaldor, N. Causes of the slowrate of economic growth in the United Kingdom, en Targetti, Thirleall, Anthony, comp. 1989. The essential Kaldor, Duckworth, London. 1966.

- Kalmanovitz, S. "La rentabilidad decreciente de la industria en Colombia", en Ensayos escogidos de economía colombiana, Bogotá, Universidad Nacional.1988.

- Lotero, J A. La industria en la región del eje cafetero, 1975 - 1990 evolución y retos futuros, Estudio presentado al seminario "Ciudadanía, democracia y desarrollo en el eje cafetero”, Manizales. Fotocopia, 1993.

- Ramírez, O. ¿Es Manizales una ciudad industrial? Tesis maestría en economía. U.N de Colombia. Bogotá. 2004.

- Serrano, E; Dussán, C. Un análisis sobre la dinámica de las PMI en Manizales 1990-2003. Informe final de investigación. VIP U. Caldas. 2007.

- Vieira, J; et al. Informe Final Diplomado en Desarrollo Económico y Competitividad. Universidad Autónoma de Manizales. Mimeo. Diciembre 15 de 2000 .

\section{NOTAS}

1. Presentada a la Vicerrectoría de Investigaciones y Postgrados de la Universidad de Caldas en 2007.

2. Encuesta de Opinión Empresarial, la entrevista realizada a expertos y la Encuesta desarrollada por el Grupo de investigación a empresarios de las Industrias representativas de Manizales entre julio y agosto de 2007.

3.Acervo de capital o capital acumulado en al industria en todos los proceso de adquisición de maquinarias y equipos.

4. Se eligió el mes de diciembre teniendo en cuenta la finalización de año y una mirada proactiva frente al siguiente año; es posible que el mes elegido sea susceptible de sesgos pero aún así los datos son una confirmación de la situación nacional y regional de la época.

5. El concepto de balance en las encuestas de opinión corresponde a la diferencia entre el porcentaje de respuestas "positivas" (mayor, aumentó, favorable, etc.) a cada pregunta y las respuestas "negativas" (menor, disminuyó, desfavorable, etc.).

6. Entrevistas a expertos locales y encuestas a empresas.

7. Entrevista a Taniguichi Kisyoshi (2006).

8. El 78\% de los entrevistados respondió que el instinto influye en estas decisiones.

9. Para Vieira et, al (9) utilizando una serie de inversión con la Encuesta de Opinión Empresarial la alta dinámica de la inversión "no se concentró en un solo año sino en los primeros cuatro de la década del noventa, en 1995 se redujo la tasa de crecimiento de la inversión, y desde 1996 se presentaron reducciones en sus niveles”. Pero Ramírez (7) encuentra con base en EAM que los años 1992 y 1995 son los años cuando la inversión tuvo un repunte pronunciado en la pasada década. 